\title{
45. MAGNETIC PROPERTIES OF BASALTS, DEEP SEA DRILLING PROJECT LEGS 69 AND 701
}

\author{
D. M. Pechersky and L. V. Tikhonov, Institute of Physics of the Earth, \\ Academy of Sciences of the U.S.S.R., Moscow, U.S.S.R. \\ and \\ N. N. Pertsev, Institute of Geology of Ore Deposits, Mineralogy, Petrography, Geochemistry, \\ Academy of Sciences of the U.S.S.R., Moscow, U.S.S.R.
}

\begin{abstract}
We studied magnetic properties of basalts from cores of Sites 504 (mainly Hole 504B) and 505. According to magnetic and petrographic data, the basalts are altered to different degrees. We determined the ratio $I_{\mathrm{ST}} / I_{\mathrm{so}}$ and the Curie points of the basalts. In Hole 504B there is vertical zoning of titanomagnetite alteration: in the upper part of the basement, low temperature titanomaghemitization predominates $\left(I_{\mathrm{ST}} / I_{\mathrm{so}}>1\right)$; in the lower part of the hole, multi-phase alteration $\left(I_{\mathrm{ST}} / I_{\mathrm{so}}<1\right)$ predominates. This is evidence for superimposition of multi-phase alteration after single-phase oxidation. The latter process occurred at low temperatures. Multi-phase alteration is represented by exsolution and unmixing of titanomagnetite. Its appearance is connected with extended secondary heating at temperatures of about $100^{\circ} \mathrm{C}$, documented by thermal measurements in the hole. Magnetic properties of basalts from Site 505 are typical of submarine pillow lavas that contain fine-grained titanomaghemite.
\end{abstract}

\section{INTRODUCTION}

We studied magnetic properties of 86 samples from Site 504 (Holes 504B and 504A), and 11 samples from Site 505. The general aim of the work was to study the structure and development of Layer 2 of the ocean crust. Our main objective in this chapter is to outline the magnetic characteristics of altered rock of different kinds in Hole 504B, the deepest hole drilled in the Costa Rica Rift area.

The following magnetic characteristics were measured: natural remanent magnetization (NRM, $\left.I_{\mathrm{n}}\right)$, magnetic susceptibility, saturation magnetization $\left(I_{\mathrm{s}}\right)$, saturation remanence $\left(I_{\mathrm{rs}}\right)$, and remanent coercive force $\left(H_{\mathrm{CR}}\right)$. Median destructive fields of NRM $\left(\mathrm{MDF}_{\mathrm{n}}\right)$ and $I_{\mathrm{rs}}$ $\left(\mathrm{MDF}_{\mathrm{s}}\right)$ were determined. Thermomagnetic analysis (thermal demagnetization of $I_{\mathrm{s}}$ and $I_{\mathrm{rs}}$ ) was carried out. Magnetic investigations were carried out at the Laboratory of the Geomagnetic Field of the Institute of Physics of the Earth, Moscow. Values of NRM and other kinds of remanent magnetization were measured with a spinner magnetometer. Measurements of $I_{\mathrm{s}}$ and its thermal demagnetization were made with a vibro-magnetometer in a constant magnetic field of 2500 Oe. Heating of all the samples was in air; the heating rate was $\sim 30^{\circ} / \mathrm{min}$. From the thermomagnetic results the ratio of the saturation magnetizations observed at room temperature after $\left(\mathrm{I}_{\mathrm{ST}}\right)$ and before $\left(\mathrm{I}_{\mathrm{so}}\right)$ heating was calculated. AFdemagnetization of $I_{\mathrm{n}}$ and $I_{\mathrm{rs}}$ was carried out with a standard device, with a maximum alternating field of 800 Oe. Susceptibility was measured with a high-frequency kappameter. The results of magnetic measurements are given in Table 1 . In Tables 2 and 3 we present

${ }^{1}$ Cann, J. R., Langseth, M. G., Honnorez, J., Von Herzen, R. P., White, S. M., et al., Init. Repts. DSDP, 69: Washington (U.S. Govt. Printing Office). mean values of the magnetic characteristics of basalts from Hole 504B.

\section{PETROGRAPHIC GROUPING AND MAGNETIC PROPERTIES OF BASALTS, HOLE 504B}

Using petrographic data, we subdivide the basalt into two types according to conditions of crystallization: pillow lavas, and massive lava flows (and/or sills). In the upper part of Hole 504B, pillow lavas and thin flows dominate; in the lower part, massive flows and sills dominate. According to degree of alteration, the basalts were divided into three groups: (1) fresh, (2) altered rocks with a background alteration, making up most of the section (the rocks are mostly light green), (3) local zones of high oxidation (red, brown, and green colors dominating). For convenience, we shall call these three groups "fresh," "green," and "red." Because "red" alteration was very local, adjacent to small cracks and narrow zones, it was impossible to study many specimens with "red" alteration only; thus, in Tables 2 and 3 "red" specimens were chosen from places where high oxidation dominates.

First, we note that titanomagnetite (TM) is altered even in "fresh" basalts. This is evident from their high Curie points $\left(T_{\mathrm{c}}\right)$, near $370^{\circ} \mathrm{C}$ (Tables 1 and 2). At the same time, using electron-microprobe analysis, Pertsev and Boronikhin (this volume) calculated a $T_{\mathrm{c}}$ change from 85 to $280^{\circ} \mathrm{C}$, with a mean $T_{\mathrm{c}}$ of $180^{\circ} \mathrm{C}(13 \mathrm{sam}$ ples). This is close to the mean $T_{\mathrm{c}}$ of primary TM of oceanic basalts (Johnson, 1979). Hence, genuinely fresh basalts are absent in Hole 504B. "Fresh," "green," and "red"' basalts differ only in the degree of alteration. In both pillows and massive basalts, $T_{\mathrm{c}}, H_{\mathrm{CR}}, I_{\mathrm{rs}} / I_{\mathrm{s}}$, and MDF increase, whereas $I_{\mathrm{n}}$ and $I_{\mathrm{s}}$ decrease as alteration increases from "fresh" to "red" basalts. That is why the degree of TM oxidation and its magnetic hardness increase. The decrease of $I_{\mathrm{n}}$ and $I_{\mathrm{s}}$ evidently is connected 


\section{M. PECHERSKY, L. V. TIKHONOV, N. N. PERTSEV}

Table 1. Magnetic properties of basalt samples, Sites 504 and 505.

\begin{tabular}{|c|c|c|c|c|c|c|c|c|c|c|}
\hline $\begin{array}{c}\text { Sample } \\
\text { (level in cm) }\end{array}$ & $x\left(10^{6} \mathrm{emu}\right)$ & $I_{\mathrm{n}}\left(10^{3} \mathrm{emu}\right)$ & $Q_{n}$ & $\begin{array}{l}H_{C R} \\
(\mathrm{Oe})\end{array}$ & $\begin{array}{l}\mathrm{MDF}_{\mathrm{n}} \\
(\mathrm{Oe})\end{array}$ & $\begin{array}{l}\mathrm{MDF}_{\mathrm{s}} \\
(\mathrm{Oe})\end{array}$ & $\begin{array}{c}I_{\mathrm{s}} \\
(\mathrm{Ganss} \\
\left.\mathrm{cm}^{3} / \mathrm{g}\right)\end{array}$ & $I_{\mathrm{rs}} / I_{\mathrm{s}}$ & $\begin{array}{l}T_{\mathrm{c}} \\
\left({ }^{\circ} \mathrm{C}\right)\end{array}$ & $I_{\mathrm{ST}} / I_{\mathrm{so}}$ \\
\hline 504B-2-1, 103 & 495 & 1.25 & 5.1 & 128 & 135 & $\begin{array}{l}78 \\
62\end{array}$ & $\begin{array}{l}0.53 \\
0.774\end{array}$ & 0.15 & $\begin{array}{r}390 \\
380\end{array}$ & 1.6 \\
\hline $\begin{array}{l}3-1,103 \\
4-1,44\end{array}$ & $\begin{array}{l}864 \\
342\end{array}$ & 10.7 & $\begin{array}{l}8.7 \\
62.6\end{array}$ & $\begin{array}{l}120 \\
222\end{array}$ & $\overline{175}$ & $\begin{array}{l}62 \\
120\end{array}$ & $\begin{array}{l}0.0774 \\
0.56\end{array}$ & $\begin{array}{l}0.15 \\
0.23\end{array}$ & $\begin{array}{l}380 \\
370\end{array}$ & 1.76 \\
\hline $4-2,23$ & 130 & 4.45 & 68.5 & 688 & - & 450 & 0.20 & 0.29 & $\begin{array}{l}370 \\
380\end{array}$ & 1.76 \\
\hline $4-3,91$ & 300 & 6.70 & 44.7 & 185 & 160 & 95 & 0.567 & 0.18 & 370 & $1 . \overline{77}$ \\
\hline $4-4,80$ & 460 & 5.70 & 24.8 & 158 & - & 95 & 0.612 & 0.16 & 360 & 1.75 \\
\hline $4-5,76$ & 127 & 3.00 & 47.2 & 430 & 300 & 250 & 0.173 & 0.22 & 400 & 1.21 \\
\hline $5-1,109$ & 131 & 1.90 & 29.0 & 647 & - & - & 0.115 & 0.17 & - & - \\
\hline $\begin{array}{l}5.2,108 \\
5,3,69\end{array}$ & $\begin{array}{l}256 \\
110\end{array}$ & $\begin{array}{l}14.60 \\
1.50\end{array}$ & 114 & ${ }_{267}^{124}$ & 102 & 65 & 0.774 & 0.19 & $\begin{array}{l}350 \\
380\end{array}$ & 1.11 \\
\hline $\begin{array}{l}5-3,69 \\
6-1,119\end{array}$ & 160 & $\begin{array}{l}9.50 \\
8.50\end{array}$ & $\begin{array}{l}119 \\
147\end{array}$ & $\begin{array}{l}267 \\
493\end{array}$ & $\overline{400}$ & $\overline{320}$ & $\begin{array}{l}0.53 \\
0.276\end{array}$ & $\begin{array}{l}0.26 \\
0.32\end{array}$ & $\begin{array}{l}380 \\
370\end{array}$ & 1.22 \\
\hline $6-2,99$ & $\begin{array}{l}4110 \\
480\end{array}$ & 4.20 & $\begin{array}{l}147.5 \\
17.5\end{array}$ & $\begin{array}{r}493 \\
93 \\
\end{array}$ & - & - & 0.877 & $\begin{array}{l}0.32 \\
0.14\end{array}$ & 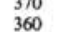 & $\begin{array}{l}1.21 \\
1.5\end{array}$ \\
\hline $6-2,122$ & 162 & 5.00 & 61.7 & 250 & 200 & 130 & 0.545 & 0.16 & $\begin{array}{l}380 \\
380\end{array}$ & 1.4 \\
\hline $7-1,45$ & 330 & 3.00 & 18.2 & 540 & - & - & 0.164 & 0.23 & - & - \\
\hline $7-2,100$ & 430 & 8.60 & 40 & 242 & 250 & 130 & 0.577 & 0.21 & 370 & 1.65 \\
\hline $7-3,45$ & 158 & 3.63 & 45.9 & 550 & - & - & 0.207 & 0.28 & 420 & 1.14 \\
\hline $7-5,71$ & 543 & 1.92 & 7.1 & 106 & 80 & 45 & 0.93 & 0.09 & 370 & 1.54 \\
\hline $\begin{array}{l}8-1,78 \\
8-2,109\end{array}$ & $\begin{array}{l}648 \\
389\end{array}$ & 2.05 & ${ }_{3.3}^{6.3}$ & 95 & $\overline{7}$ & $\overline{75}$ & 0.869 & 0.11 & 350 & 1.75 \\
\hline $\begin{array}{l}8-2,199 \\
9-2,93\end{array}$ & $\begin{array}{l}389 \\
215\end{array}$ & $\begin{array}{l}0.62 \\
6.36\end{array}$ & $\begin{array}{r}3.2 \\
59.2\end{array}$ & $\begin{array}{l}1177 \\
216\end{array}$ & 170 & ${ }_{-}^{75}$ & $\begin{array}{l}0.771 \\
0.042\end{array}$ & $\begin{array}{l}0.12 \\
0.21\end{array}$ & $\begin{array}{l}370 \\
370\end{array}$ & 1.52 \\
\hline $10-3,109$ & 139 & $\begin{array}{l}.350 \\
1.50\end{array}$ & 21.6 & $\begin{array}{l}610 \\
614\end{array}$ & 375 & $\overline{400}$ & 0.0412 & & 370 & 1.16 \\
\hline $11-2,2$ & 412 & 3.81 & 18.5 & 180 & - & - & 0.807 & 0.19 & 390 & $\overline{1.61}$ \\
\hline $12-2,33$ & 390 & 4.54 & 23.3 & 226 & 175 & 120 & 0.663 & 0.18 & 370 & $\begin{array}{l}1.60 \\
1.65\end{array}$ \\
\hline $13-4,10$ & 813 & 2.51 & 6.2 & 100 & - & - & 0.843 & 0.14 & 365 & 1.52 \\
\hline $13-4,145$ & 700 & 1.32 & ${ }_{78}^{3.7}$ & 130 & 120 & 65 & 0.739 & 0.15 & 380 & 1.46 \\
\hline $\begin{array}{l}14-2,91 \\
15.4\end{array}$ & $\begin{array}{l}254 \\
254\end{array}$ & 9.91 & $\begin{array}{l}78 \\
65\end{array}$ & $\begin{array}{l}212 \\
225\end{array}$ & $\overline{195}$ & $\overline{105}$ & $\begin{array}{l}0.599 \\
0.36 \%\end{array}$ & 0.23 & $\begin{array}{l}380 \\
375\end{array}$ & 1.0 \\
\hline $\begin{array}{l}15-4,73 \\
15-5,87\end{array}$ & $\begin{array}{l}248 \\
800\end{array}$ & $\begin{array}{l}8.0 \\
1.13\end{array}$ & $\begin{array}{l}65 \\
2.8\end{array}$ & $\begin{array}{l}235 \\
123\end{array}$ & $\begin{array}{l}195 \\
115\end{array}$ & $\begin{array}{l}125 \\
63\end{array}$ & $\begin{array}{l}0.396 \\
0.803\end{array}$ & 0.22 & $\begin{array}{l}375 \\
365\end{array}$ & $\begin{array}{l}0.97 \\
1.43\end{array}$ \\
\hline $16-2,25$ & 600 & 1.87 & 6.2 & 129 & - & - & 0.882 & 0.14 & $\begin{array}{l}503 \\
360\end{array}$ & $\begin{array}{l}1.43 \\
1.52\end{array}$ \\
\hline $16-3,52$ & 780 & 3.64 & 9.3 & 130 & - & - & 1.044 & 0.15 & 380 & 1.58 \\
\hline $16-4,35$ & 180 & 2.9 & 32.2 & 298 & 280 & 187 & 0.237 & 0.23 & 395 & -0 \\
\hline $16-4,54$ & 314 & 4.65 & 29.6 & 185 & - & - & 0.458 & 0.21 & 400 & 0.93 \\
\hline $17-1,2$ & 440 & 5.88 & 26.7 & 234 & 190 & 125 & 0.528 & 0.20 & - & - \\
\hline $17-2,72$ & $\begin{array}{l}520 \\
286\end{array}$ & 2.60 & 10 & 185 & - & $\overline{0}$ & 0.538 & 0.15 & 360 & 1.11 \\
\hline $\begin{array}{l}18 \cdot 2,4 \\
192,106\end{array}$ & $\begin{array}{l}286 \\
675\end{array}$ & $\begin{array}{l}5.25 \\
4.66\end{array}$ & $\begin{array}{l}36.7 \\
13.8\end{array}$ & ${ }_{213}^{185}$ & 150 & 98 & $\begin{array}{l}0.566 \\
0.42\end{array}$ & $\begin{array}{l}0.25 \\
0.20\end{array}$ & $\begin{array}{l}360 \\
360\end{array}$ & $\begin{array}{l}1.82 \\
0.95\end{array}$ \\
\hline $20-1,84$ & 600 & $\begin{array}{l}4.66 \\
1.37\end{array}$ & $\begin{array}{r}13.8 \\
4.6\end{array}$ & $\begin{array}{l}213 \\
102\end{array}$ & $\overline{145}$ & $\overline{85}$ & $\begin{array}{l}0.442 \\
0.835\end{array}$ & $\begin{array}{l}0.20 \\
0.11\end{array}$ & $\begin{array}{l}360 \\
360\end{array}$ & $\begin{array}{l}0.95 \\
1.63\end{array}$ \\
\hline $21-1,30$ & 52 & 5.10 & 196 & 295 & - & - & 0.433 & 0.23 & 395 & $\begin{array}{l}1.63 \\
1.42\end{array}$ \\
\hline $21 \cdot 2,104$ & 380 & 4.40 & 23.2 & 152 & 145 & 78 & 0.720 & 0.15 & 380 & 1.17 \\
\hline $21-3,12$ & 82 & 1.74 & 42.4 & 405 & - & - & 0.241 & 0.24 & - & - \\
\hline $21-4,3$ & 520 & 4.40 & 17 & 120 & 90 & 60 & 0.769 & 0.15 & 380 & 1.68 \\
\hline $\begin{array}{l}21 \cdot 5,3 \\
22,3\end{array}$ & 182 & 2.16 & 23.7 & $\begin{aligned} 229 \\
225\end{aligned}$ & 175 & 150 & 0.310 & 0.19 & 400 & 0.9 \\
\hline $\begin{array}{l}22-1,34 \\
23-1,113\end{array}$ & $\begin{array}{l}315 \\
660\end{array}$ & $\begin{array}{l}4.61 \\
3.68\end{array}$ & 29.3 & $\begin{array}{l}225 \\
174\end{array}$ & $\overline{140}$ & $\overline{89}$ & 0.645 & 0.18 & 390 & -7 \\
\hline $\begin{array}{l}23-1,113 \\
24-1,88\end{array}$ & $\begin{array}{l}630 \\
320\end{array}$ & $\begin{array}{l}3.68 \\
5.33\end{array}$ & $\begin{array}{l}11.7 \\
33.3\end{array}$ & $\begin{array}{l}174 \\
225\end{array}$ & 140 & 89 & $\begin{array}{l}0.954 \\
0.484\end{array}$ & $\begin{array}{l}0.16 \\
0.23\end{array}$ & $\begin{array}{l}380 \\
400 \\
400\end{array}$ & $\begin{array}{l}1.28 \\
0.91\end{array}$ \\
\hline $25-1,34$ & 213 & 6.56 & 61.3 & 212 & 155 & 121 & 0.475 & 0.24 & $\begin{array}{l}400 \\
390\end{array}$ & $\begin{array}{l}0.91 \\
0.93\end{array}$ \\
\hline $25-2,113$ & 60 & 4.0 & 133 & 289 & - & - & 0.298 & 0.23 & - & 0.93 \\
\hline $26-1,3$ & 1130 & 1.71 & 3.0 & 120 & 95 & 56 & 1.220 & 0.12 & 385 & 1.38 \\
\hline $27-2,87$ & 340 & 1.94 & 11.4 & 193 & - & $\overline{0}$ & 0.592 & 0.19 & 370 & 1.42 \\
\hline $28-1,32$ & 230 & 0.96 & 8.3 & 408 & 300 & 216 & 0.154 & 0.20 & 520 & 0.88 \\
\hline $28-1,90$ & 1180 & 1.85 & 3.1 & 67 & - & - & 1.126 & 0.07 & 365 & 1.25 \\
\hline $\begin{array}{l}28-3,93 \\
28.4\end{array}$ & 650 & 2.00 & 6.2 & 132 & $\overline{7}$ & $\overline{2}$ & 0.884 & 0.14 & 390 & 1.32 \\
\hline $\begin{array}{l}28-4,15 \\
29-1,27\end{array}$ & $\begin{array}{l}67 \\
105\end{array}$ & $\begin{array}{l}2.47 \\
5.74\end{array}$ & $\begin{array}{l}74.8 \\
590\end{array}$ & $\begin{array}{l}365 \\
{ }_{102}\end{array}$ & 270 & 212 & $\begin{array}{l}0.276 \\
0.088\end{array}$ & 0.25 & $\begin{array}{l}400 \\
400\end{array}$ & 0.96 \\
\hline $\begin{array}{l}29-1,27 \\
32-2,18\end{array}$ & 195 & 5.74 & 39.0 & $\begin{array}{l}192 \\
196\end{array}$ & $\bar{z}$ & $\overline{-}$ & $\begin{array}{l}0.068 \\
0.680\end{array}$ & $\begin{array}{l}0.16 \\
0.20\end{array}$ & $\begin{array}{l}400 \\
410 \\
410\end{array}$ & $\begin{array}{l}0.83 \\
0.91\end{array}$ \\
\hline $33-1,94$ & 1267 & - & $\overline{-}$ & 108 & $=$ & $=$ & 0.756 & 0.14 & $\begin{array}{l}410 \\
380\end{array}$ & $\begin{array}{l}0.91 \\
1.70\end{array}$ \\
\hline $34-1,63$ & 649 & - & - & 242 & - & - & 0.570 & 0.20 & 420 & 1.02 \\
\hline $34-2,18$ & 328 & - & - & 340 & - & - & 0.215 & 0.21 & 400 & 0.95 \\
\hline $35-1,96$ & 459 & - & - & 265 & - & - & 0.443 & 0.18 & 390 & 1.23 \\
\hline $36-1,88$ & 1241 & - & - & 97 & - & - & 1.253 & 0.08 & 315 & 0.89 \\
\hline $\begin{array}{l}36-3,110 \\
37-3,18\end{array}$ & 1189 & - & - & ${ }^{70}$ & - & - & 1.190 & 0.06 & 290 & 0.86 \\
\hline $\begin{array}{l}37-3,18 \\
41-1,38\end{array}$ & $\begin{array}{r}1100 \\
987\end{array}$ & $\bar{z}$ & $=$ & $\begin{array}{r}125 \\
60\end{array}$ & $\bar{z}$ & $\bar{z}$ & $\begin{array}{l}0.729 \\
0.900\end{array}$ & $\begin{array}{l}0.15 \\
0.06\end{array}$ & $\begin{array}{l}410 \\
{ }_{280}\end{array}$ & 1.62 \\
\hline $\begin{array}{l}41-1,38 \\
42-1,32\end{array}$ & $\begin{array}{l}987 \\
1948\end{array}$ & $\overline{-}$ & $=$ & $\begin{array}{l}60 \\
68\end{array}$ & $=$ & $\bar{z}$ & $\begin{array}{l}0.900 \\
1.317\end{array}$ & $\begin{array}{l}0.06 \\
0.05\end{array}$ & $\begin{array}{l}280 \\
415 \\
415\end{array}$ & $\begin{array}{l}0.89 \\
1.10\end{array}$ \\
\hline $44-2,65$ & 1345 & - & - & 80 & - & - & 1.100 & 0.09 & 405 & $\begin{array}{l}1.10 \\
0.92\end{array}$ \\
\hline $46-1,70$ & 1196 & - & - & 127 & - & - & 1.006 & 0.13 & 435 & 0.80 \\
\hline $46-3,106$ & 632 & - & - & 180 & - & - & 0.578 & 0.15 & 430 & 0.78 \\
\hline $47-2,20$ & 1177 & - & - & 120 & - & - & 1.072 & 0.10 & 385 & 0.89 \\
\hline $\begin{array}{l}47-3,36 \\
4\end{array}$ & 1715 & - & - & 80 & - & - & 1.237 & 0.05 & 425 & 1.10 \\
\hline $\begin{array}{l}48-3.142 \\
49-2,85\end{array}$ & 1244 & $=$ & $=$ & ${ }_{107}^{148}$ & $\bar{z}+$ & $\overline{-}$ & 0.971 & 0.15 & 420 & 0.72 \\
\hline $\begin{array}{l}49-2,85 \\
51-1,15\end{array}$ & $\begin{array}{l}1604 \\
1091\end{array}$ & $=$ & $=$ & $\begin{array}{r}107 \\
62\end{array}$ & $\overline{-}$ & $\overline{-}$ & $\begin{array}{l}\begin{array}{l}1.160 \\
1.062\end{array} \\
\end{array}$ & $\begin{array}{l}0.10 \\
0.07\end{array}$ & $\begin{array}{l}440 \\
310\end{array}$ & $\begin{array}{l}0.90 \\
0.85\end{array}$ \\
\hline $53-1,40$ & 1952 & - & - & 78 & - & - & $\begin{array}{l}1.062 \\
1.415\end{array}$ & 0.06 & $\begin{array}{l}310 \\
460\end{array}$ & $\begin{array}{l}0.85 \\
0.78\end{array}$ \\
\hline $56-2,66$ & 1605 & - & - & 90 & - & - & 1.216 & 0.07 & 420 & 0.79 \\
\hline $57-3,105$ & 1631 & - & - & 73 & - & - & 1.318 & 0.06 & 375 & 1.00 \\
\hline $60-2,56$ & 1000 & - & - & 121 & - & - & 1.068 & 0.17 & 400 & 0.71 \\
\hline $62-1,122$ & 615 & - & - & 287 & - & - & 0.656 & 0.23 & 455 & 0.65 \\
\hline $\begin{array}{l}63-3,3,15 \\
642,28\end{array}$ & 1692 & - & - & 93 & - & - & 1.098 & 0.13 & 445 & 0.70 \\
\hline $\begin{array}{l}64-2,28 \\
64,4,3\end{array}$ & $\begin{array}{l}2153 \\
2287\end{array}$ & $\bar{z}$ & $=$ & $\begin{array}{l}86 \\
87\end{array}$ & $\bar{z}+$ & $\bar{z}$ & 1.714 & 0.07 & $\begin{array}{l}475 \\
4700\end{array}$ & 0.86 \\
\hline $\begin{array}{l}64-4,3 \\
66-2,56\end{array}$ & $\begin{array}{l}2287 \\
2734\end{array}$ & $=$ & $=$ & $\begin{array}{l}87 \\
93\end{array}$ & $=$ & $=$ & $\begin{array}{l}1.478 \\
1.926\end{array}$ & 0.04 & $\begin{array}{l}470 \\
490\end{array}$ & $\begin{array}{l}0.87 \\
0.90\end{array}$ \\
\hline $70-1,86$ & 1325 & - & - & 146 & - & - & $\begin{array}{l}1.1920 \\
1.186\end{array}$ & 0.03 & 500 & 0.90 \\
\hline $504 \mathrm{~A} 6-1,17$ & 984 & 11.49 & 23.4 & 75 & 68 & 42 & 1.220 & 0.12 & 300 & 0.91 \\
\hline $\begin{array}{l}6-2,75 \\
6-3.85\end{array}$ & $\begin{array}{l}1092 \\
900\end{array}$ & $\begin{array}{l}4.14 \\
2.96\end{array}$ & $\begin{array}{l}7.6 \\
6.6\end{array}$ & $\begin{array}{l}90 \\
95\end{array}$ & 75 & 45 & 0.954 & 0.14 & 370 & \\
\hline $\begin{array}{r}6-3,85 \\
505 \mathrm{~A}-1-1,36\end{array}$ & 900 & 2.96 & 6.6 & 95 & 106 & 52 & 0.785 & 0.16 & 370 & 1.76 \\
\hline $\begin{array}{c}505 \mathrm{~A}-1-1,36 \\
2-1,5\end{array}$ & $\begin{array}{l}39 \\
42\end{array}$ & $\begin{array}{r}2.35 \\
11.53\end{array}$ & $\begin{array}{l}120 \\
549\end{array}$ & $\begin{array}{r}1135 \\
780\end{array}$ & $\overline{600}$ & $\overline{525}$ & $\begin{array}{l}0.121 \\
0.268\end{array}$ & $\begin{array}{l}0.30 \\
0.43\end{array}$ & $\begin{array}{l}380 \\
340\end{array}$ & $\begin{array}{l}1.46 \\
2.25\end{array}$ \\
\hline $505 \mathrm{~B}-1-1,3$ & 24 & 10.07 & 839 & 610 & 555 & 400 & 0.271 & 0.45 & 350 & 2.46 \\
\hline $2-1,129$ & 40 & 5.69 & 285 & 415 & 360 & 255 & 0.296 & 0.38 & 320 & 2.22 \\
\hline $2-2,119$ & 39 & 9.78 & 489 & 700 & - & - & 0.218 & 0.42 & 320 & 1.96 \\
\hline $2-2,135$ & 14 & 12.70 & 1814 & 400 & 375 & 260 & 0.306 & 0.42 & 280 & 1.99 \\
\hline $2-3,18$ & 14 & 3.26 & 466 & 270 & 248 & 150 & 0.368 & 0.24 & 340 & 1.97 \\
\hline 3-1, & 54 & 9.12 & 338 & 617 & 500 & 600 & 0.220 & 0.41 & 320 & 1.88 \\
\hline 3-2, 115 & 23 & 5.07 & 441 & 337 & 355 & 200 & 0.308 & $\begin{array}{l}0.36 \\
0.30\end{array}$ & 280 & 1.99 \\
\hline $\begin{array}{l}5-1,27 \\
6-1,97\end{array}$ & ${ }_{22}^{20}$ & $\begin{array}{r}10.39 \\
8.39\end{array}$ & $\begin{array}{l}1039 \\
763\end{array}$ & $\begin{array}{l}250 \\
170\end{array}$ & $\begin{array}{l}225 \\
400\end{array}$ & $\begin{array}{l}150 \\
120\end{array}$ & $\begin{array}{l}0.043 \\
0.519\end{array}$ & $\begin{array}{l}0.30 \\
0.26\end{array}$ & $\begin{array}{l}230 \\
230 \\
250\end{array}$ & 1.16 \\
\hline
\end{tabular}


Table 2. Average magnetic characteristics of pillow basalts, Hole 504B.

\begin{tabular}{lccccc}
\hline $\begin{array}{c}\text { Magnetic } \\
\text { Characteristic }\end{array}$ & $\begin{array}{c}\text { Leg 69 } \\
\text { (5 samples) }\end{array}$ & $\begin{array}{c}\text { "Green," } \\
\text { Leg 69 } \\
\text { (13 samples) }\end{array}$ & $\begin{array}{c}\text { "Red," } \\
\text { Leg 69 } \\
\text { (13 samples) }\end{array}$ & $\begin{array}{c}\text { "Red," } \\
\text { Leg 70 } \\
\text { (18 samples) }\end{array}$ & $\begin{array}{c}\text { All } \\
\text { Samples }\end{array}$ \\
\hline $\begin{array}{l}\text { (106) } \\
I_{\mathrm{n}}\left(10^{3} \text { ) }\right.\end{array}$ & 307 & 300 & 318 & - & - \\
$H_{\mathrm{CR}}$ & 4.39 & 6.58 & 3.65 & - & - \\
$I_{\mathrm{S}}$ & 230 & 304 & - & - & 394 \\
$I_{\mathrm{TS}} / I_{\mathrm{S}}$ & 0.54 & 0.445 & 0.276 & 0.53 & 0.36 \\
$T_{\mathrm{c}}$ & 0.204 & 0.219 & - & - & 0.176 \\
$I_{\mathrm{ST}} / I_{\mathrm{SO}}$ & 384 & 379 & - & - & 440 \\
$\mathrm{MDF}_{\mathrm{n}}$ & 1.2 & 1.13 & - & - & 1.21 \\
$\mathrm{MDF}_{\mathrm{S}}$ & 200 & 195 & 207 & - & - \\
\hline
\end{tabular}

with the decrease of TM magnetic moment during single-phase oxidation. It is weaker in massive basalts.

As seen in Tables 1 to 3, there are no principal differences between "fresh," "'green," and "red" samples according to their magnetic properties. Consequently, at the level of our data, both pillows and massive rocks from "fresh" to "red" differ only in their degrees of alteration. These differences are lesser in massive basalts (Tables 2 and 3). "Red" rocks dominate in the upper part of the section to approximately Core 40 . In this upper part of the basement, single-phase oxidation obviously dominates both in pillows and basalt flows. We shall return to this question later.

As a rule, basalts in the upper part of the section are from thin flows, but in their conditions of crystallization they differ from pillow lavas. This is reflected in the fact that $H_{\mathrm{CR}}, I_{\mathrm{rs}} / I_{\mathrm{s}}$, and MDF are lower, whereas $\chi$ and $I_{\mathrm{s}}$ are higher than in pillow lavas. However, the degree of single-phase oxidation is higher in flows $\left(I_{\mathrm{ST}} / I_{\mathrm{so}}\right.$ is higher; Tables 2 and 3).

\section{THERMOMAGNETIC INVESTIGATION OF BASALT ALTERATION, HOLE 504B}

Thermomagnetic-analysis data $I_{\mathrm{s}}(\mathrm{T})$ show that TM single-phase oxidation occurred at low temperature in the upper part of Hole 504B, where pillow and thin flow lavas dominate (Fig. 1A). In the process of heating titanomaghemite (TMH) destructs and magnetite appears. The cooling curve runs much higher than the heating curve. Thermomagnetic-analysis curves $I_{\mathrm{s}}(\mathrm{T})$ are different in the lower part of Hole 504B, where massive flows are more common (Fig. 1B). During heating, magnetite does not appear, and the second heating curve is lower than the first $\left(I_{S T} / I_{s o}<1\right) ; T_{\mathrm{c}}$ is shifted to low temper- atures. The shift is greater the higher the initial Curie point is. The $I_{\mathrm{s}}(\mathrm{T})$ curves become practically reversible during repeated heating of such samples to $600^{\circ} \mathrm{C}$, and only long heating at 500 to $600^{\circ} \mathrm{C}$ leads to a picture typical of high-temperature TM multi-phase oxidation, where $I_{\mathrm{s}}$ increases and a new phase with $T_{\mathrm{c}}$ close to that of magnetite appears.

Analogous results were obtained in massive basalts cored during Leg 58 (Furuta et al., 1980) and Leg 65 (Pechersky et al., in press a, b). This behavior of $I_{\mathrm{s}}$ during heating is explained by rehomogenization of previously unmixed TM grains (Pechersky et al., in press a, b). Because the process takes place in air at rather low temperature $\left(<600^{\circ} \mathrm{C}\right)$, we supposed that the unmixing structure is very fine. A similar magnetic effect was observed in the case of TM oxidized in the laboratory at $T$ $<200^{\circ} \mathrm{C}$ (Johnson and Merrill, 1973). It was explained by those authors as a rehomogenization effect. Samples from Leg 65 had a distinct $T_{\mathrm{c}}<200^{\circ} \mathrm{C}$ after heating, which is close to $T_{\mathrm{c}}$ of primary TM. Based on microprobe data, the mean calculated $T_{\mathrm{c}}=155^{\circ} \mathrm{C}$ in TM grains of basalts from Leg 65 (Pechersky et al., in press b). But samples from Hole 504B have no distinct $T_{\mathrm{c}}$ after heating (Fig. 1B).

In a number of cases $I_{\mathrm{s}}(\overline{\mathrm{T}})$ is of intermediate character (Fig. $1 \mathrm{~A}-\mathrm{C}$ ). The $I_{\mathrm{s}}(\mathrm{T})$ of the first heating resembles typical initial heating curves for TMH, having a bump at temperatures higher than 300 to $400^{\circ} \mathrm{C}$; however, after heating $I_{\mathrm{s}}$ does not increase, and even decreases, and upon reheating $T_{\mathrm{c}}$ shifts downward (Fig. 1C).

Being aware that primary TM in basalts from Hole $504 \mathrm{~B}$ as a rule has $100^{\circ} \mathrm{C} \leq T_{\mathrm{c}} \leq 200^{\circ} \mathrm{C}$, we can use the ratio $I_{\mathrm{ST}} / I_{\mathrm{so}}$ as a sign of predominance of single-phase (Fig. 1A) or multi-phase (Fig. 1B) types of TM alteration. $I_{\mathrm{ST}} / I_{\mathrm{so}}<1$ is evidence for a predominance of multiphase oxidation (unmixing). There is a marked correlation between $I_{\mathrm{ST}} / \mathrm{I}_{\mathrm{so}}$ and $T_{\mathrm{c}}$ (Fig. 2). The figure shows the predominance of unmixing in samples with $T_{\mathrm{c}} \geq$ $400^{\circ} \mathrm{C}$. In cases of complete predominance of singlephase oxidation $\left(I_{\mathrm{ST}} / I_{\mathrm{so}}>1.4\right), T_{\mathrm{c}} \leq 400^{\circ} \mathrm{C}$ (Fig. 2). This coincides with the data of Readman and O'Reilly (1972), that for TMH $(Z=1)$ which formed from TM with $T_{\mathrm{c}}$ $=200^{\circ} \mathrm{C}, T_{\mathrm{c}} \simeq 370^{\circ} \mathrm{C}$.

We do not preclude unmixing in samples with $T_{\mathrm{c}}<$ $400^{\circ} \mathrm{C}$. For example, a group of massive-basalts samples with $T_{\mathrm{c}}=250$ to $320^{\circ} \mathrm{C}$ have $I_{\mathrm{ST}} / I_{\mathrm{so}}<0.9$ (Fig. 2). There may be both TMH and TM grains in the same sample. Unmixing is more distinct in the case of pillow lavas.

Table 3. Average magnetic characteristics of flow basalts, Hole 504B.

\begin{tabular}{|c|c|c|c|c|c|c|c|c|}
\hline $\begin{array}{c}\text { Magnetic } \\
\text { Characteristics }\end{array}$ & $\begin{array}{l}\text { "Fresh," } \\
\text { Leg } 69 \\
\text { (5 samples) }\end{array}$ & $\begin{array}{c}\text { "Fresh," } \\
\text { Leg } 70 \\
\text { (5 samples) }\end{array}$ & $\begin{array}{c}\text { "Fresh," } \\
\text { All } \\
\text { Samples } \\
\text { (10 samples) }\end{array}$ & $\begin{array}{l}\text { "Green," } \\
\text { Leg } 69\end{array}$ & $\begin{array}{l}\text { “Green," } \\
\text { Leg } 70\end{array}$ & $\begin{array}{c}\text { "'Green," } \\
\text { All } \\
\text { Samples } \\
\text { (24 samples) }\end{array}$ & $\begin{array}{c}\text { "Red," } \\
\text { Leg } 69 \\
\text { (13 samples) }\end{array}$ & $\begin{array}{c}\text { "Red," } \\
\text { Leg } 70 \\
\text { (3 samples) }\end{array}$ \\
\hline$x\left(10^{6}\right)$ & 417 & - & - & 409 & - & - & 552 & 1266 \\
\hline$I_{\mathrm{n}}\left(10^{3}\right)$ & 3.82 & - & - & 3.82 & - & - & 2.66 & - \\
\hline$H_{C R}$ & 162 & 87 & 125 & 189 & 116 & 140 & 162 & 124 \\
\hline$I_{\mathrm{S}}$ & 0.687 & 1.117 & 0.902 & 0.665 & 1.119 & 1.014 & 0.845 & 0.918 \\
\hline$I_{\mathrm{rS}} / I_{\mathrm{S}}$ & 0.152 & 0.076 & 0.114 & 0.14 & 0.11 & 0.12 & 0.145 & 0.13 \\
\hline$T_{\mathrm{c}}$ & 377 & 350 & 363 & 373 & 433 & 413 & 374 & 400 \\
\hline$I_{\mathrm{ST}} / I_{\mathrm{SO}}$ & 1.57 & 0.91 & 1.24 & 1.33 & 0.82 & 0.98 & 1.57 & 1.24 \\
\hline $\mathrm{MDF}_{\mathrm{n}}$ & 140 & - & - & 172 & - & - & 159 & - \\
\hline $\mathrm{MDF}_{\mathrm{s}}$ & 80 & - & - & 101 & - & - & 98 & - \\
\hline
\end{tabular}



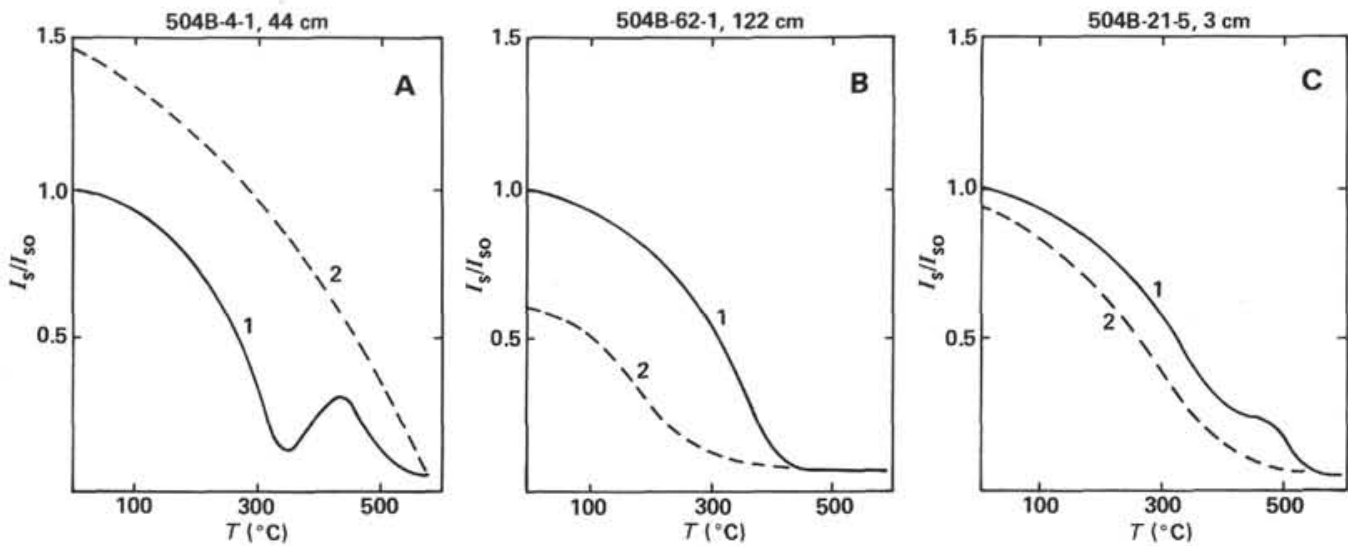

Figure 1. Examples of thermomagnetic-analysis curves. A. Titanomaghemite. B. Multi-phase alteration of titanomagnetite (exsolution predominates). C. Intermediate case. 1-first heating; 2-second heating.

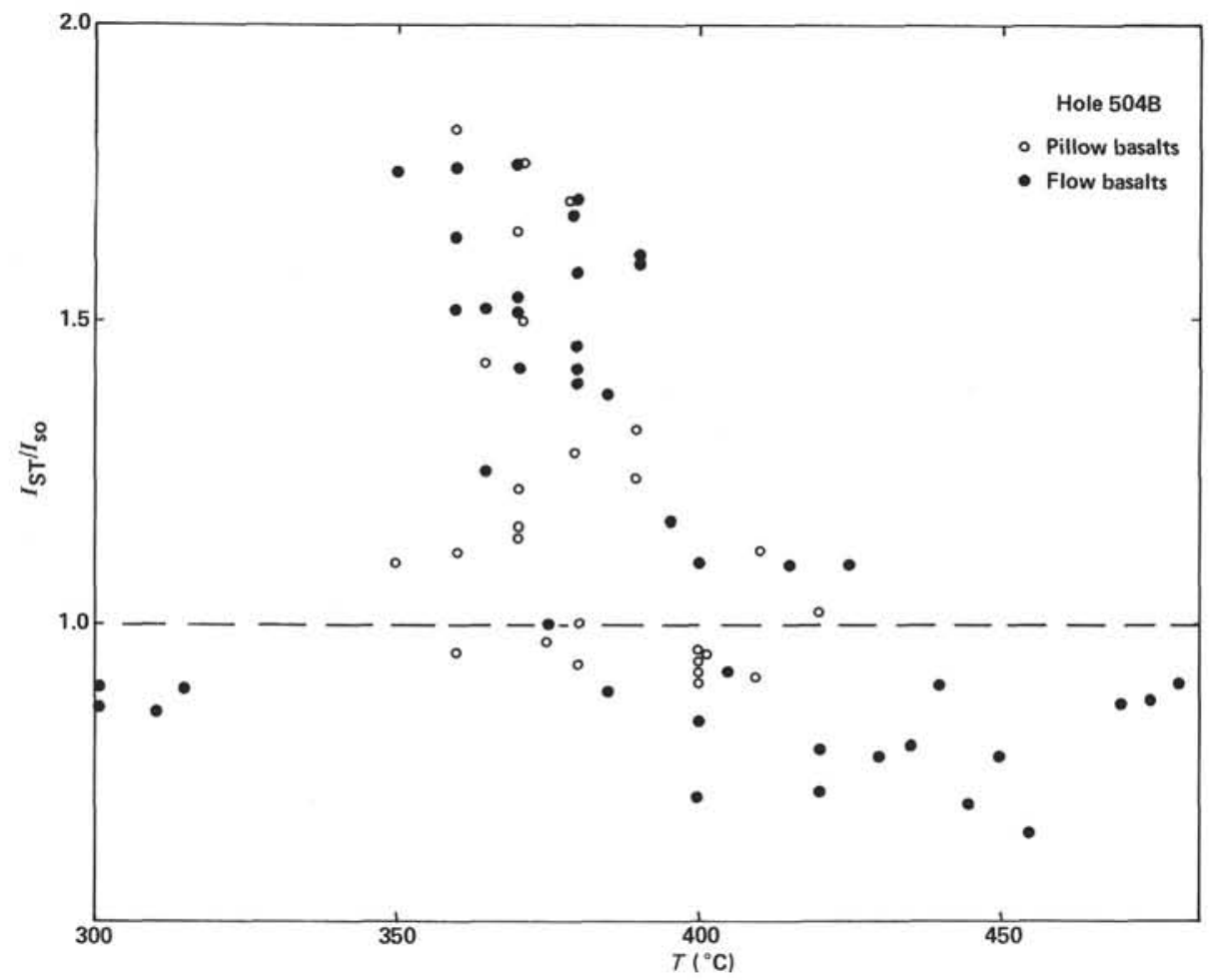

Figure 2. Relation between $I_{\mathrm{ST}} / I_{\mathrm{so}}$ and Curie points.

Such samples are characterized by intermediate $I_{\mathrm{s}}(\mathrm{T})$; $I_{\mathrm{ST}} / I_{\mathrm{so}} \sim 0.9$ to $1.2 ; T_{\mathrm{c}}=350$ to $420^{\circ} \mathrm{C}$. They occupy intermediate positions in Figure 2.

\section{COMPARISON OF PETROGRAPHIC AND THERMOMAGNETIC DATA WITH THE DEGREE OF ROCK OXIDATION}

The predominance of single-phase TM oxidation in basalts from Hole 504B $\left(I_{\mathrm{ST}} / I_{\mathrm{so}}>1\right)$ correlates with petrographic data; namely, in all examined thin-sections of basalt samples with $I_{\mathrm{ST}} / I_{\mathrm{so}}>1$, for both pillow and massive lavas, there are marked signs of strong low-temperature oxidation, such as iron hydroxides in veins and opaque grains, brown oxidized glass, red-brown, and green clay minerals, etc. These signs of low-temperature oxidation are absent in samples with $I_{\mathrm{ST}} / I_{\mathrm{so}}<1$ and $I_{s}(\mathrm{~T})$, as in the sample shown in Figure 1B. In a single sample (504B-15-4, 73-76 cm), which has $I_{s}(T)$ of intermediate type (Fig. 1C), there are places affected by "red" oxidation and places without signs of such oxidation. In other samples (504B-33-1, 94-97 cm) of similar magnetic type, sulfides(?) are remarkable oxidized, but TM grains have no petrographic signs of oxidation.

\section{VERTICAL ZONING OF TM ALTERATION IN HOLE 504B}

The two main types of TM alteration are rather regularly distributed throughout Hole 504B. $T_{\mathrm{c}}$ increases with 
depth (Fig. 3A), but $I_{\mathrm{ST}} / I_{\mathrm{so}}$ decreases (Fig. 3B). $I_{\mathrm{ST}} / I_{\mathrm{so}}$ for pillow lavas occurs at the depth of Core 14, but for massive basalts it occurs at Core 35 . The zone of predominance of unmixed TM $\left(T_{\mathrm{c}}>400^{\circ} \mathrm{C} ; I_{\mathrm{ST}} / I_{\mathrm{so}}<1\right)$ is situated below Core 45. The gradual change in TM alteration can be seen more distinctly if $I_{\mathrm{ST}} / I_{\mathrm{so}}$ values are averaged at five-core intervals (Fig. 3C).

Thus, two types of TM alteration are discerned in magnetic properties of basalts. Their distribution is regular along the hole. A similar vertical zoning was noticed for Hole 418A (Fig. 3D).

We relate TM unmixing to post-magmatic hydrothermal processes which heat the rocks. For instance, in the bottom of Hole 504B the temperature is $110^{\circ} \mathrm{C}$ (Becker et al., this volume). In the bottom of Hole 482B (Leg 65) the temperature is $80^{\circ} \mathrm{C}$ (Robinson, Lewis et al., in press). In the latter case, widespread TM unmixing has also occurred (Pechersky et al., in press a, b). In rocks of Hole $418 \mathrm{~A}$ heating above $40^{\circ} \mathrm{C}$ is suggested by mineralogical data (Pertsev and Rusinov, 1980).

\section{HEATING-COOLING CYCLES OF SAMPLES WITH $I_{\mathrm{ST}} / I_{\mathrm{so}}<1$}

Experiments involving stepped heating and maintenance at fixed temperatures, for example on Sample 504B-62-1, 122-124 cm (Fig. 4), show that a decrease in $I_{\mathrm{s}}$ takes place above $300^{\circ} \mathrm{C}$. Further heating to $400^{\circ} \mathrm{C}$, and maintenance there for 5 min leads to a remarkable drop in $I_{\mathrm{s}}$, the shape of the $I_{\mathrm{s}}(\mathrm{T})$ curve and $T_{\mathrm{c}}$ value being invariant. After heating to $500^{\circ} \mathrm{C}, I_{\mathrm{s}}$ decreases and the $I_{\mathrm{s}}(\mathrm{T})$ curve becomes concave. A distinct, fixed $T_{\mathrm{c}}$ is not observed. It is possible that high-temperature unmixing is simultaneous with rehomogenization or that rehomogenization was not complete. A partly concave $I_{s}(\mathrm{~T})$ curve for the final heating may be explained by variations in the composition of primary TM, as shown by microprobe data on some samples (Pertsev and Boronikhin, this volume; Furuta, this volume).

Why $I_{\mathrm{s}}$ falls but $T_{\mathrm{c}}$ does not change after heating to $400^{\circ} \mathrm{C}$ is not clear to us yet.

\section{MAGNETIC PROPERTIES OF BASALTS OF SITE 505}

Basalts of Site 505 differ from those of Site 504 in their magnetic properties (Table 1). Their mean values are $I_{\text {n }}$ $=7.64 \times 10^{-3} \mathrm{emu} ; I_{\mathrm{rs}} / I_{\mathrm{s}}=0.356 ; H_{\mathrm{CR}}=502 \mathrm{Oe} ; I_{\mathrm{ST}} /$ $I_{\mathrm{so}}=1.94 ; I_{\mathrm{s}}=0.294 \mathrm{Gauss} \cdot \mathrm{cm}^{3} / \mathrm{g}$; and $T_{\mathrm{c}}=320^{\circ} \mathrm{C}$. These magnetic characteristics are typical of oceanic pillow lavas and evidence for a predominance of finegrained TMH.

\section{CONCLUSIONS}

Lately, discovered peculiarities in magnetic properties of some oceanic basalts are not typical of oceanic basalts with TMH. These are high Curie points of 300 to $500^{\circ} \mathrm{C}, I_{\mathrm{ST}} / I_{\mathrm{so}}<1$, and shift of $T_{\mathrm{c}}$ at the second heating (or cooling) to the left, approaching the Curie point of primary TM of oceanic basalts. The primary TM composition is similar in all oceanic basalts, with $T_{\mathrm{c}} \approx 150^{\circ} \mathrm{C}$. This type of alteration is widespread in basalts of Hole 504B.

The observed magnetic peculiarities more often are found in massive-basalts samples, but they also occur in pillow basalts, for instance, in Hole 504B. In samples with such magnetic peculiarities, TM grains with ilmenite exsolution lamellae often occur, i.e., features of unmixing (Legs 51-53, 58, 65). In a number of cases the unmixing can be explained by high-temperature oxidation of TM during slow cooling of massive flows (e.g., Genkin et al., 1980; Pechersky et al., in press b). However, in these cases there are typically large ilmenite exsolution lamellae in TM, but TM grains in Hole 504B
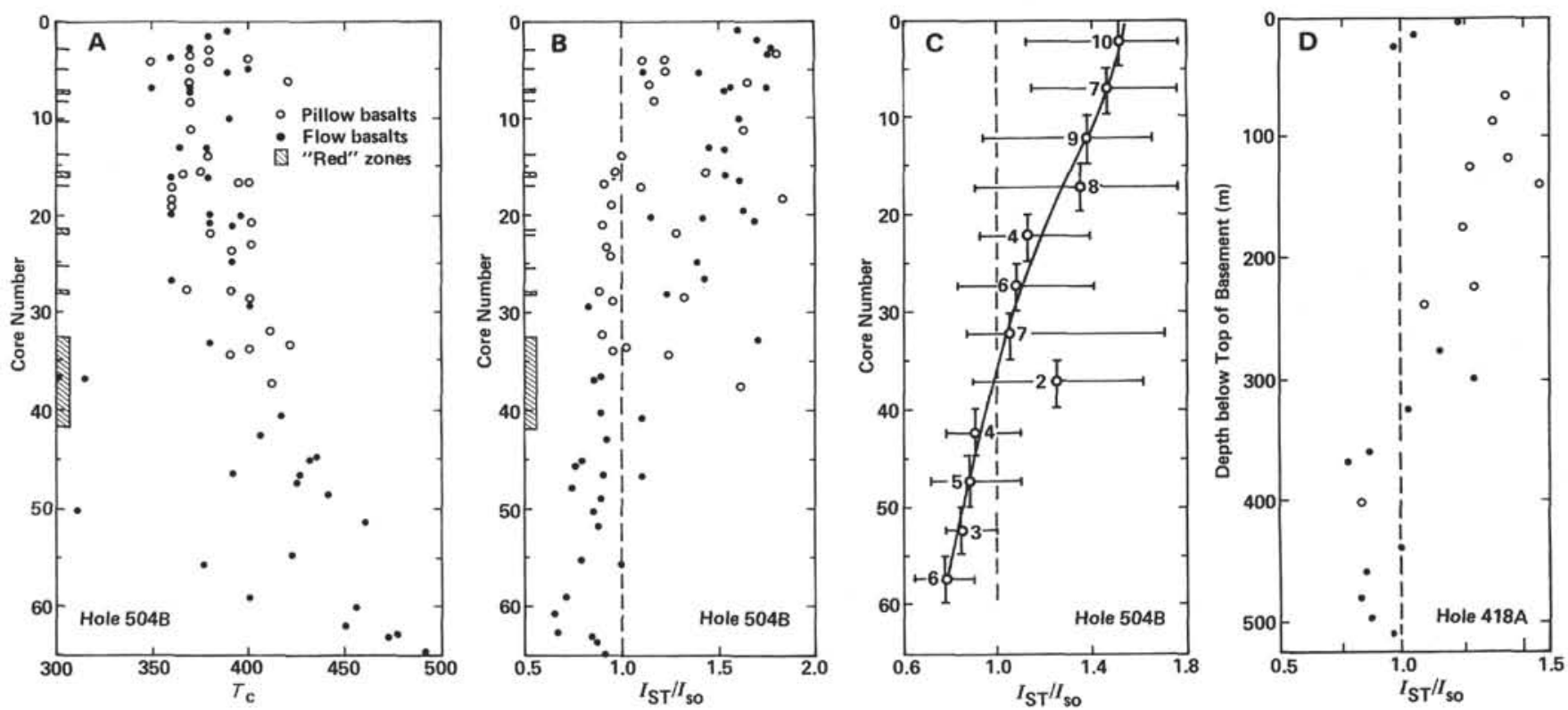

Figure 3. Distribution of magnetic characteristics with depth for Holes $504 \mathrm{~B}$ and $418 \mathrm{~A}$. A. Curie points. B. The ratios $I_{\mathrm{ST}} / I_{\mathrm{so}}$. C. Mean values of $I_{\mathrm{ST}} / I_{\mathrm{so}}$ for five core intervals; figures near the circles represent number of samples. D. $I_{\mathrm{ST}} / I_{\mathrm{so}}$ for Hole $418 \mathrm{~A}$. 


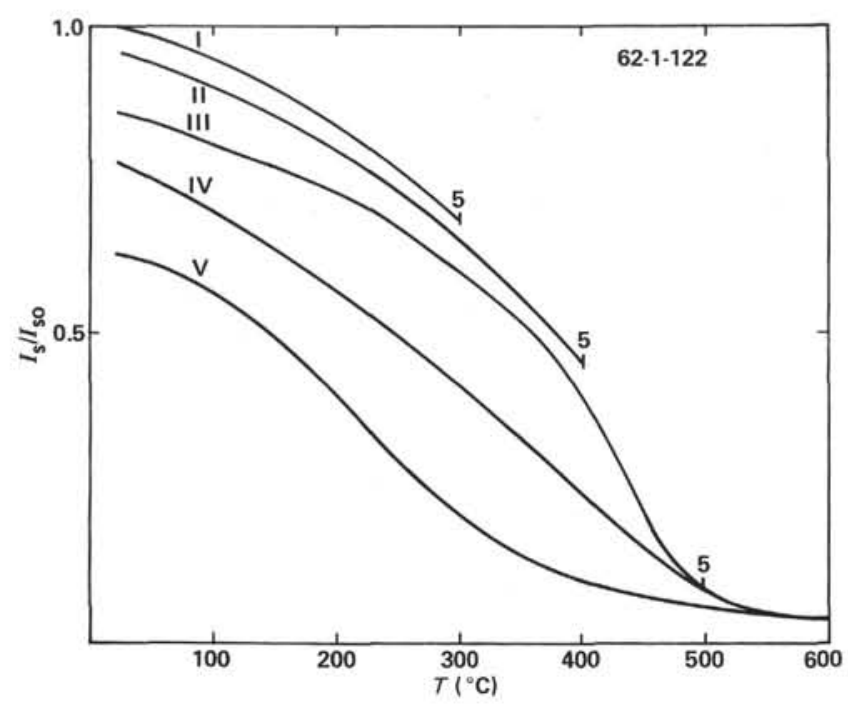

Figure 4. Example of $I_{\mathrm{s}}$ changes during heating-cooling cycles of one sample. Figures near the curves-the time of heating in minutes at the fixed temperature. $\mathrm{I}-\mathrm{Y}-$ cycles of heating.

basalts have a fine structure of unmixing and exsolution (Pechersky et al., in press b). We relate this fine unmixing of TM to low-temperature alteration during long secondary heating of the rocks. The following are arguments in favor of this explanation:

1) There is a regular appearance of high Curie points coupled with $I_{\mathrm{ST}} / I_{\mathrm{so}}<1$ and the disappearance of "red" zones with depth in Holes 504B and 418A;

2) These unusual magnetic properties appear in basalts from Holes with anomalously high temperatures, and in basalts exhibiting hydrothermal alterations $(T<$ $200^{\circ} \mathrm{C}$ );

3) Rehomogenization of $\mathrm{TM}$ at about $500^{\circ} \mathrm{C}$ contradicts high-temperature unmixing;

4) The presence of these magnetic properties-not only in massive flows, but also in pillow lavas-contradicts high-temperature alteration of TM;

5) According to the solvus diagram of Basta (1960), TM with $T_{\mathrm{c}} \approx 400^{\circ} \mathrm{C}$ forms as result of exsolution at $T<200^{\circ} \mathrm{C}$.

The occurrence in individual core sections, and even in individual samples, of TMH grains and unmixed TM grains is evidence for superimposition of unmixing on low-temperature oxidation. A process of single-phase oxidation producing lamellae of ulvospinel or ilmenite in grains of unmixed TM is unlikely. It is natural that during extended oxidation of such grains further unmixing without TMH formation will occur. Thus, superimposition of unmixing on pre-existing alteration that produced TMH is a strong argument that the latter process took place at low temperature $\left(<200^{\circ} \mathrm{C}\right)$.

There has not yet been an experimental test of our explanation of the phenomenon in question. In all known experiments during heating of TM and TMH there appear magnetic minerals with $T_{\mathrm{c}}>500^{\circ} \mathrm{C}$, whereas basalts with supposed low-temperature TM unmixing have $T_{\mathrm{c}}<450^{\circ} \mathrm{C}$.

\section{ACKNOWLEDGMENTS}

We are thankful to V. N. Vadkovsky and G. Z. Gurary for careful reading of the manuscript, discussion, and both critical and useful remarks.

\section{REFERENCES}

Basta, E., 1960. Natural and synthetic titanomagnetites (the system $\mathrm{Fe}_{3} \mathrm{O}_{4}-\mathrm{Fe}_{2} \mathrm{TiO}_{4}-\mathrm{FeTiO}_{3}$ ). Neues Jahrb. Mineral., 94:1017-1048.

Burakov, K. S., 1977. Thermomagnetometer. Izv. AN SSSR, Ser. Fizika Zemli, 5:92-96.

Furuta, T., Kobayashi, K., and Momose K., 1980. Magnetic properties of igneous rocks of the Philippine sea, Deep Sea Drilling Project Leg 58. In Kobayashi, K., Klein, G. deV., et al., Init. Repts. DSDP, 58: Washington (U.S. Govt. Printing Office), 923-934.

Genkin, A. D., Laputina, I. P., and Pertsev, N. N., 1980. Opaque minerals in basalts from Holes 417D and 418A. In Donnelly, T., Francheteau, J., Bryan, W., Robinson, P., Flower, M., Salisbury, M., et al., Init. Repts. DSDP, 51, 52, 53, Pt. 2: Washington (U.S. Govt. Printing Office), 1431-1450.

Johnson, H. P., 1979. Magnetization of the oceanic crust. Rev. Geophys. Space Phys., 17:215-226.

Johnson, H. P., and Merrill, R. T., 1973. Low-temperature oxidation of a titanomagnetite and the implications for paleomagnetism. $J$. Geophys. Res., 78:4938-4949.

Pechersky, D. M., Tikhonov, L. V., and Zolotarev, B. P., in press a. Magnetism and paleomagnetism of basalts, DSDP Leg 65. In Robinson, R., Lewis, B., et al., Init. Repts. DSDP, 65: Washington (U.S. Govt. Printing Office).

, in press b. Magnetism and paleomagnetism of basalts, Gulf of California (Leg 65 of Glomar Challenger). Izv. AN SSSR, Ser. Fizika Zemli.

Pertsev, N. N., and Rusinov, V. L., 1980. Mineral assemblages and processes of alteration in basalts at Deep Sea Drilling Project Sites 417 and 418. In Donnelly, T., Francheteau, J., Bryan, W., Robinson, P., Flower, M., Salisbury, M., et al., Init. Repts. DSDP, 51, 52, 53, Pt. 2: Washington (U.S. Govt. Printing Office), 1219-1242.

Readman, P., and O'Reilly W., 1972. Magnetic properties of oxidized (cation-deficient) titanomagnetites $(\mathrm{Fe}, \mathrm{Ti},)_{3} \mathrm{O}_{4} . J$. Geomagnet. Geoelec., 24:69-90.

Robinson, P., Lewis, B., et al., in press. Init. Repts. DSDP, 65: Washington (U.S. Govt. Printing Office). 